\title{
Edema pulmonar agudo no cardiogénico secundario a hidroclorotiazida. Reporte de un caso y revisión de la literatura
}

\author{
JAVIERA VENEGAS B.*, CRISTÓBAL MARAMBIO C.*, \\ MARÍA GUACOLDA BENAVIDES G.** y CARLOS INZUNZA P.***
}

\section{Hydrochlorothiazide induced non-cardiogenic acute pulmonary edema. A case report}

Thiazides are drugs often used in management of high arterial blood pressure. Shock and acute pulmonary edema are rarely described as adverse reactions related to this drug. We report the case of a 55 years-old woman admitted at Hospital de Puerto Montt, Chile. Two hours after having her first dose of hydrochlorothiazide she presented dyspnea. Laboratory tests and images support the diagnosis of non-cardiogenic pulmonary edema. Resolution of her clinical picture was observed 48 hours after hydrochlorothiazide administration was discontinued and hemodynamic and non invasive ventilation support were supplied. The patient was discharged without symptoms, 3 days after entering to hospital.

Key words: Pulmonary Edema; Hydrochlorothiazide; Pharmaceutical preparations; Non invasive ventilation.

\section{Resumen}

Las tiazidas son fármacos frecuentemente usados en la terapia de la hipertensión arterial. Las reacciones adversas de riesgo vital como shock y edema pulmonar agudo son raros. Comunicamos el caso de una mujer de 55 años de edad atendida en Hospital de Puerto Montt, quien tras dos horas de ingerir hidroclorotiazida presentó disnea. Los exámenes de laboratorio generales e imágenes muestran cuadro concordante con edema pulmonar agudo no cardiogénico. Además de la suspensión del fármaco, se realizó soporte hemodinámico y ventilatorio no invasivo, evidenciándose resolución del cuadro a las 48 h. La paciente fue dada de alta 3 días después de su ingreso sin sintomatología.

Palabras clave: Edema pulmonar; hidroclorotiazida; preparados farmacéuticos; ventilación no invasiva.

\section{Introducción}

El edema agudo de pulmón representa una patología de gran importancia clínica debido a su alta mortalidad, reportada entre un $33-41 \%$. Fisiopatológicamente, esta entidad puede ser clasificada como $^{2}$ :

- Causa cardiogénica: originada por aumento de la presión hidrostática capilar pulmonar, debido a una elevación de presión en la aurícula izquierda;
- Causa no cardiogénica (acute lung injury o acute respiratory distress): originada por un aumento de la permeabilidad de los capilares pulmonares.

El edema pulmonar agudo no cardiogénico fue definido clínicamente en 1994 por la Conferencia de Consenso Americano Europeo, donde se estableció como criterio un cuadro de inicio agudo, además de un infiltrado bilateral a la radiografía de tórax; y una presión de arteria pulmonar me-

* Estudiantes de Medicina, Universidad de Los Andes.

** Médico Broncopulmonar, Clínica Universidad de Los Andes.

*** Médico Broncopulmonar, Hospital de Puerto Montt. 
nor a $18 \mathrm{mmHg}$, o evidencia clínica de que no existe hipertensión en aurícula izquierda. Se definieron como criterios de gravedad una $\mathrm{PaO}_{2} / \mathrm{FiO}_{2}$ $\leq 300 \mathrm{mmHg}$ para ALI (acute lung injury) y una $\mathrm{PaO}_{2} / \mathrm{FiO}_{2} \leq 200$ para ARDS (acute respiratory distress síndrome) $)^{3}$.

Se ha reportado que alrededor del 3\% de los casos de edema pulmonar no cardiogénico están asociados a la administración de medicamentos ${ }^{1}$. La mayoría de estos episodios corresponde a reacciones idiosincráticas, independientes de la dosis y el tiempo de administración del fármaco. Por lo anterior, no está claramente establecida la fisiopatología que lo desencadena.

Existe una amplia lista de fármacos con casos reportados de edema pulmonar agudo no cardiogénico. Destacan aquellos medicamentos con más de 10 publicaciones al respecto, entre ellos, metadona, naloxona, salbutamol e hidroclorotiazida ${ }^{4}$.

El edema pulmonar agudo no cardiogénico secundario al uso de hidroclorotiazida es un cuadro poco prevalente, ya que solo existen alrededor de 50 casos publicados en la literatura médica ${ }^{5}$. No obstante, su importancia radica en el frecuente uso de este medicamento en el control de la hipertensión arterial.

A continuación, se presenta un caso clínico compatible con edema agudo pulmonar inducido por hidroclorotiazida.

\section{Descripción del caso}

Paciente de 55 años, sexo femenino, con antecedente de Diabetes mellitus tipo 2 no insulino requiriente en tratamiento con metformina/glibenclamida, hipertensión arterial e insuficiencia cardiaca capacidad funcional I-II, en tratamiento con losartán $100 \mathrm{mg} /$ día. La paciente acudió a control de sus comorbilidades en donde se le indicó cambio de losartán por Hidroronol T (hidroclorotiazida $25 \mathrm{mg}+$ triamterene $50 \mathrm{mg}$ ), del cual ingiere $1 / 2$ comprimido durante la consulta.

A las $2 \mathrm{~h}$, la paciente presenta un cuadro de dolor abdominal difuso, vómitos y disnea progresiva, por lo que decide consultar en servicio de urgencia de Frutillar. Ingresa taquicárdica 140 lpm, hipertensa 205/124 $\mathrm{mmHg}$, polipneica 25 ciclos por min y desaturando hasta $53 \%$ de oxihemoglobina con aire ambiental. Se sospecha edema pulmonar agudo y se maneja con furosemida, morfina, propanolol, hidrocortisona, adenosina y nebulización con berodual. Se indica traslado al Hospital de Puerto Montt.

Ingresa al hospital de Puerto Montt taquicárdica $(132 \mathrm{lpm})$, hipotensa $(75 / 56 \mathrm{mmHg})$, polipneica y saturando $88 \%$ con $\mathrm{FiO}_{2}=1$, afebril. Examen pulmonar muestra murmullo pulmonar disminuido con crépitaciones escasas bilaterales y edema de extremidades inferiores leve, bilateral. Resto del examen se encuentra normal.

Se toman los siguientes exámenes en servicio de urgencia: $\mathrm{pH}: 7,291, \mathrm{PaO}_{2} 63,7 \mathrm{mmHg}\left(\mathrm{PaO}_{2} /\right.$ $\mathrm{FiO}_{2}=63,7 \mathrm{mmHg}$ ), hemoglobina $16,2 \mathrm{~g} / \mathrm{dL}$, Leucocitos $10.600 / \mathrm{mm}^{3}$ (recuento diferencial normal), Plaquetas $401.000 / \mathrm{mm}^{3}$, PCR $6 \mathrm{mg}$ / dL (VN: $<10 \mathrm{mg} / \mathrm{dL}$ ), Creatinina 1,24 mg/dL. Troponina T, CKMB, electrolitos plasmáticos y unción hepática dentro de rangos normales. Por la procedencia rural de la paciente, se realiza test para Hanta virus, el cual resulta negativo. El electrocardiograma muestra una taquicardia sinusal de $125 \mathrm{lpm}$, sin signos de isquemia.

Se solicita tomografía computada de tórax que descarta un tromboembolismo pulmonar y muestra múltiples opacidades en vidrio esmerilado confluentes, engrosamiento intersticial y escaso derrame pleural bilateral (Figura 1).

La paciente es trasladada a UCI para apoyo ventilatorio con VMNI (ventilación mecánica no invasiva) y tratamiento depletivo, con los siguientes signos vitales: $\mathrm{PaO}_{2}: 63,7 \mathrm{mmHg}$, $\mathrm{FiO}_{2}=1$ y $\mathrm{PaO}_{2} / \mathrm{FiO}_{2}=63,7 \mathrm{mmHg}$, desaturando hasta $53 \%$. Se maneja inicialmente como cuadro infeccioso, el cual se suspende a los 2 días por resultados negativos de pancultivos realizados al ingreso. Al segundo día de hospitalización se realiza ecocardiograma transtorácico que muestra hipertrofia del ventrículo izquierdo con función sistólica normal, leve disfunción diastólica, dilatación moderada de aurícula izquierda y leve insuficiencia mitral.

La paciente evolucionó favorablemente con resolución rápida de la falla respiratoria, hemodinámicamente estable y afebril: $\mathrm{PaO}_{2}: 64$ $\mathrm{mmHg}, \mathrm{FiO}_{2}: 0,25$ y $\mathrm{PaO}_{2} / \mathrm{FiO}_{2}=256 \mathrm{mmHg}$ con oxigenoterapia por mascarilla. Luego de $48 \mathrm{~h}$ de su ingreso, se traslada a unidad de menor complejidad $\left(\mathrm{PaO}_{2}: 73,5 \mathrm{mmHg}, \mathrm{PaO}_{2} / \mathrm{FiO}_{2}=262\right.$ $\mathrm{mmHg}, \mathrm{FiO}_{2}$ : 0,28, saturación 94\%, respirando aire ambiental), finalmente es dada de alta al día siguiente con completa resolución del cuadro clínico y normalización de los signos vitales (presión arterial: 128/65 mmHg, frecuencia cardíaca: $93 \mathrm{lpm}$, frecuencia respiratoria: $18 \mathrm{ciclos} / \mathrm{min}$ y temperatura corporal: $37,1^{\circ} \mathrm{C}$ ).

\section{Discusión}

El desarrollo de edema pulmonar no cardiogénico asociado a fármacos es poco frecuente. 


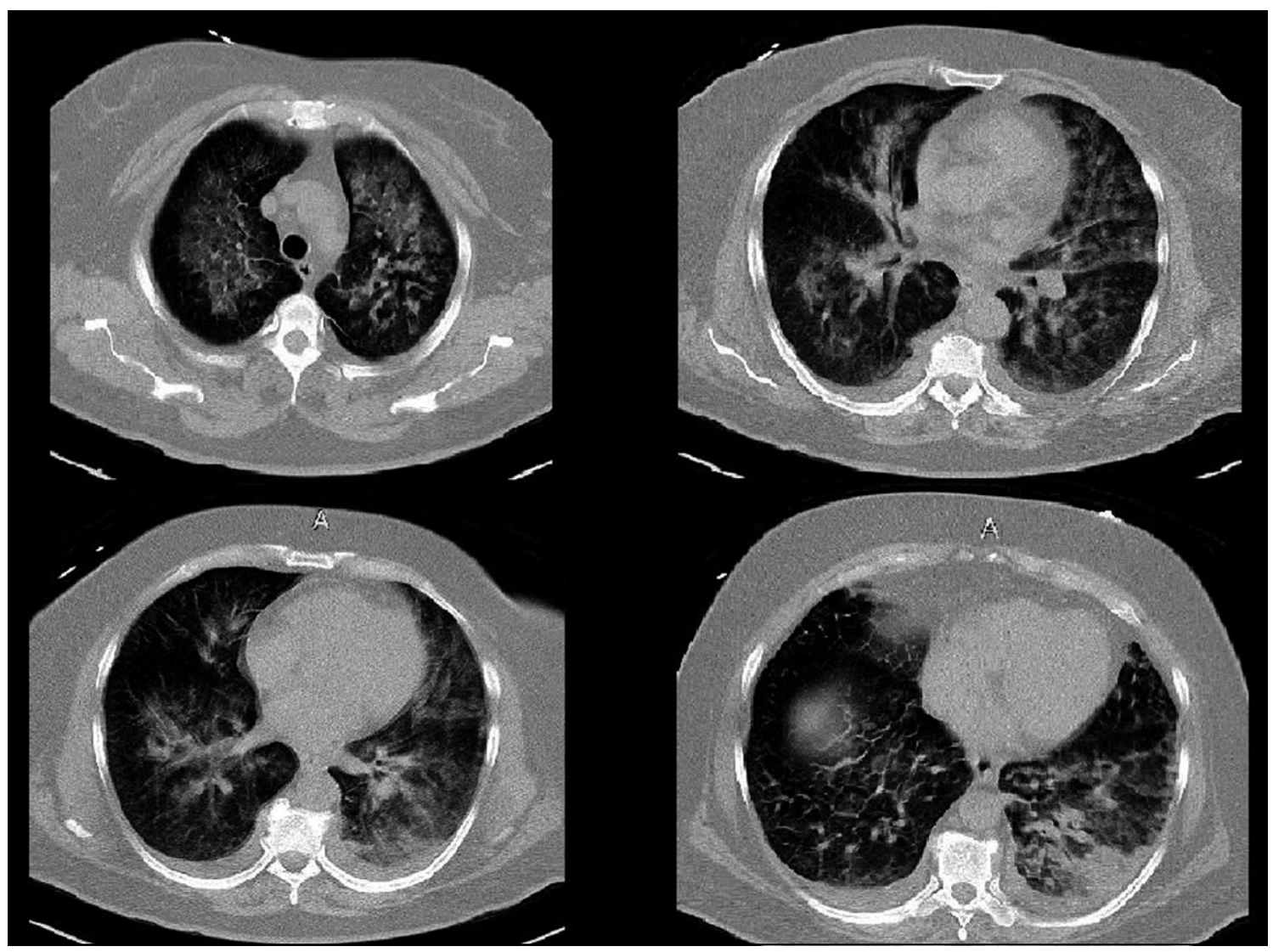

Figura 1. Tomografía computada de tórax al ingreso (ver descripción en el texto).

Los reportes señalan menos de 5\% de los casos ${ }^{6}$. Dentro de los fármacos que se han asociado a este síndrome, la hidroclorotiazida es uno de ellos. Su importancia radica en que no sólo es un fármaco de amplio uso en el tratamiento de la hipertensión arterial por su bajo costo y efectividad, sino porque también este efecto adverso puede suceder con dosis terapéuticas, a diferencia de otros fármacos como algunos opiodes y tocoliticos, en el que el sindrome se desencadena con dosis tóxicas ${ }^{3}$.

Los eventos adversos por hidroclorotiazida más frecuentes son hiponatremia, hipokalemia, hiperuricemia, hipertrigliceridemia y azoemia. Por lo anterior, el edema pulmonar no cardiogénico por hidroclorotiazida es muy infrecuente, de gravedad variable y mortalidad en el $6 \%$ de los casos $^{7}$. Una de las explicaciones al respecto es porque sus síntomas y signos son muy inespecíficos, por lo cual pocas veces se puede establecer relación causal con un fármaco determinado, y en otros, es posible que exista disfunción cardíaca asociada.

En la literatura, tanto internacional como nacional, se señala que el primer reporte de Edema pulmonar agudo no cardiogénico por tiazida data de 1968, y hasta la fecha actual se han descrito poco menos de $50 \operatorname{casos}^{5}$. La mayoría de los reportes descritos involucran a pacientes de sexo femenino (aproximadamente un 90\%) entre los 40-50 años de edad ${ }^{6}$. Puede desencadenarse tras la primera dosis de 25 a $50 \mathrm{mg}$, o en pacientes que ya habían usado este tratamiento sin presentar síntomas. También se han descrito casos de recurrencia, siendo potencialmente más graves ${ }^{8}$.

La etiopatogenia de este síndrome no es totalmente conocida, aunque se han propuesto dos mecanismos en base a los reportes de caso. La primera proposición plantea una reacción de hipersensiblidad tipo I por la presencia de eosinófilos en el lavado broncoalveolar y niveles aumentados de IgE plasmáticos, aunque sin manifestaciones de urticaria, anafilaxia o angioedema ${ }^{9}$.

Otro reporte de caso plantea una reacción de hipersensibilidad tipo III en el que se generaría IgG anti-hidroclorotiazida que se depositaría en los capilares pulmonares, generando el edema ${ }^{10}$.

Tal como se mencionó anteriormente, el cuadro clínico es muy inespecífico, caracterizado por disnea, dolor abdominal, náuseas, vómitos, fiebre 
en algunos casos, hasta la falla respiratoria aguda evidente ${ }^{3,11}$.

Los exámenes de sangre frecuentemente son normales; se ha descrito hemoconcentración en pacientes que cursaron con diarrea, leucocitosis en los que evolucionan con fiebre y más infrecuentemente trombocitopenia ${ }^{12}$.

En relación a los estudios imagenológicos, su utilidad no es tan sólo para apoyar el diagnóstico, sino también para descartar otros diagnósticos diferenciales, como embolía pulmonar y neumonía grave.

En la radiología torácica, se evidencian opacidades alvéolo-intersticiales difusas y bilaterales. En la TAC de tórax se observan opacidades en vidrio esmerilado con patrón de edema pulmonar difuso.

No obstante, lo más importante en el estudio etiológico es descartar un factor cardiogénico, dado que éste es el principal causante de edema pulmonar agudo. Es de importancia entonces, evaluar con electrocardiograma, enzimas cardiacas y eventualmente ecocardiograma transtorácico. Esto con el objetivo de descartar signos de isquemia miocárdica, o confirmar función sistólica conservada, sin signos de hipokinesia $\mathrm{y} / \mathrm{o}$ defectos valvulares que indiquen insuficiencia cardíaca como causa del cuadro clínico ${ }^{13}$.

En relación al tratamiento, no existe terapia específica, estando el manejo basado en la suspensión inmediata del fármaco y el manejo general del edema pulmonar agudo, tal como oxigenoterapia, soporte hemodinámico y respiratorio. Un tercio de los casos requiere ventilación mecánica, no invasiva o invasiva según el caso ${ }^{7}$.

No se ha demostrado que el uso de corticoides cambie la evolución ni el pronóstico ${ }^{14}$.

La resolución del cuadro clínico puede ser tan rápido como en $24 \mathrm{~h}$. La duración promedio del cuadro que se describe en la literatura es de $3,5 \pm 0,6$ días $^{15}$. Sin embargo, hay reportes que señalan que el gradiente alvéolo-arterial de oxígeno puede persistir aumentado por más de 30 días sin implicancia clínica ${ }^{11}$. En nuestro caso clínico reportado, hubo mejoría de su falla respiratoria y desaparición del patrón de relleno alveolar radiológico en el promedio de tiempo señalado.

\section{Bibliografía}

1.- RUBENFELD G D, CALDWELL E, PEABODY E, WEAVER J, MARTIN D P, NEFF M, et al. Incidence and outcomes of acute lung injury. N Engl J Med 2005; 353: 1685-93.

2.- WARE L B, MATTHAY M A. Acute pulmonary edema. N Engl J Med. 2005; 353: 2788-96.
3.- GORDON R B, ARTIGAS A, BRIGHAM K, CARLET J, FALKE K, HUDSON L, et al. The American-European consensus conference on ARDS. Am J Respir Crit Care Med 1994; 149: 818-24.

4.- REED C R, GLAUSER F L. Drug-induced Noncardiogenic Pulmonary Edema. Chest 1991; 100: 1120-4.

5.- GOETSCHALCKX K, CEUPPENS J, VAN MIEGHEM W. Hydrochlorothiazide-associated noncardiogenic pulmonary oedema and shock. Acta Cardiol 2007; 62: 215-20.

6.- KNOWLES S R, WONG G A, RAHIM S A, BINKLEY K, PHILLIPS E J, SHEAR N H. Hydrochlorothiazideinduced noncardiogenic pulmonary edema: an underrecognized yet serious adverse drug reaction, Pharmacotherapy 2005; 25: 1258-65.

7.- $\quad$ BIRON P, DESSUREAULT J, NAPKE E. Acute allergic interstitial pneumonitis induced by hydrochlorothiazide. Can Med Assoc J 1991; 145: 28-34.

8.- MAS A, JORDANA R, VALLÉS J, CERVANTES M. Recurrent hydrochlorothiazide-induced pulmonary edema. Intensive Care Med 1998; 24: 363-5.

9.- MANSO S, HEILI M, FERNÁNDEZ-NIETO B, SASTRE J. Basophil activation in two cases of hydrochlorothiazide-induced noncardiogenic pulmonary edema. Allergy 2010; 65: 135-6.

10.- BERNAL C, PATARCA R. Hydrochlorothiazideinduced pulmonary edema and associated immunologic changes. An Pharmacother 1999; 33: 172-4.

11.- GOETSCHALCKK K, CEUPPENS J, VAN MIEGHEM W. Hydrochlorothiazide-associated noncardiogenic pulmonary edema and shock: a case report and review of the literature. Act Cardiol 2007; 62: 215-20.

12.- PRUPAS HM, BROWN D. Acute idiosyncratic reaction to hydrochlorothiazide ingestion. West J Med 1983; 138: 101-2.

13.- NAYER J, AGGARWAL P, GALWANKAR S. Utility of point-of-care testing of natriuretic peptides (brain natriuretic peptide and n-terminal pro-brain natriuretic peptide) in the emergency department. Int J Chron obstruct Pulmon Dis 2014; 9: 155-62.

14.- ANDRESEN M, GONZÁLEZ A, ESPINO A, MERCADO M, REGUEIRA T, DOUGNAC A. Edema pulmonar agudo por uso de tiazidas, ¿existe falla cardíaca?: Comunicación de un caso. Rev Med Chile 2007; 4: 496-500.

15.- FINE S R, LODHA A, ZONERAICH S, MOLLURA J L. Hydro-chlorothiazide induced acute pulmonary edema. Ann Pharmacother 1995; 29: 701-3.

Correspondencia a:

Dra. María Guacolda Benavides G.

Clínica Universidad de Los Andes.

Email:mguaco@vtr.net 